KYUNGPOOK Math. J. 54(2014), 165-171

http://dx.doi.org/10.5666/KMJ.2014.54.2.165

\title{
Chain Recurrences on Conservative Dynamics
}

JAEYOO CHOY*

Department of Mathematics, Kyungpook National University, Sankyuk-dong, Bukgu, Daegu 702-701, Republic of Korea

e-mail : choy@knu.ac.kr

HAHNG-YUN CHU ${ }^{\dagger}$

Department of Mathematics, Chungnam National University, 99 Daehak-ro, YuseongGu, Daejeon 305-764, Republic of Korea

e-mail : hychu@cnu.ac.kr

Abstract. Let $M$ be a manifold with a volume form $\omega$ and $f: M \rightarrow M$ be a diffeomorphism of class $\mathrm{C}^{1}$ that preserves $\omega$. We prove that if $M$ is almost bounded for the diffeomorphism $f$, then $M$ is chain recurrent. Moreover, we get that Lagrange stable volume-preserving manifolds are also chain recurrent.

\section{Introduction}

Our purpose of this paper is to study the chain recurrence set of volumepreserving diffeomorphisms on non-compact manifolds. We follow Conley's definitions of attractors and chain recurrences [4], and Hurley's generalized definitions $[5],[6]$.

From Poincaré recurrence theorem, it is well-known that for any volumepreserving diffeomorphism on the compact manifolds $M$, every point of $M$ is chain recurrent. However, unfortunately, the parallel statement for the chain recurrence does not hold for the non-compact manifolds. Thus, in the non-compact case, we may impose the canonical conditions as almost boundedness and Lagrange stability. Our main theorem is as follows.

\footnotetext{
* Corresponding Author.

$\dagger$ The first author.

Received January 27, 2014; accepted May 22, 2014.

2010 Mathematics Subject Classification: primary 37C50; secondary 37C05, 37C70.

Key words and phrases: volume-preserving, chain recurrence, almost unbounded, Lagrange-stable, attractors.

This research was supported by Kyungpook National University Research Fund, 2013.

The second named author was supported by Basic Science Research Program through the National Research Foundation of Korea(NRF) funded by the Ministry of Education, Science and Technology(2010-0013784).
} 
Theorem 2.9. Let $M$ be a manifold with a volume form $\omega$ and $f$ be a volumepreserving diffeomorphism on $M$. If $M$ is almost bounded for $f$, then $M$ is strongly chain recurrent for $f$, i.e., every point of $M$ is strongly chain recurrent with respect to $f$.

The above theorem follows from a stronger claim, Proposition 2.10. The proposition asserts that with the assumptions in Theorem 2.9 except the Lagrange-stability assumption, almost every point in $U-A$ should have the unbounded orbit, where $A$ is an attractor and $U$ is an attractor block (weakly absorbing set) of $A$. I.e., the set of points of $U-A$ with bounded orbits is of measure 0 .

The study of attractors in the volume-preserving category is meaningful only in the non-compact cases. This is because, for a compact conservative dynamics (i.e., volume-preserving or symplectic dynamics on compact manifolds), there are only trivial attractors, which is clear from the definition of conservative diffeomorphisms (volume-preserving diffeomorphisms or symplectomorphisms on compact manifolds) [3].

Hence, we attempt to understand the volume-preserving and the symplectic dynamics on non-compact spaces through the attractors and then the chain recurrence. Whilst, similar dynamical properties on compact spaces have been intensively studied with appropriate assumptions, e.g. $\mathcal{C}^{1}$-genericity (ref.[1]). Note that since the symplectic diffeomorphisms are automatically volume-preserving, our results in the paper is immediately applicable to the symplectic dynamics as well.

\section{Chain Recurrences of Volume-preserving Diffeomorphisms}

\subsection{Preliminaries}

We fix the notations and definitions used throughout the paper.

Let $M$ be an $n$-dimensional differentiable manifold with a metric $d$, and $f: M \rightarrow$

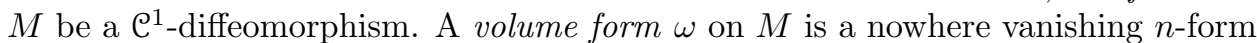
on $M$. A symplectic form $\omega$ on $M$ is a nowhere degenerate 2 -form on $M$. Here, the non-degeneracy of $\omega$ is the same as its $(n / 2)$-times wedge product $\omega^{\frac{n}{2}}=\omega \wedge \cdots \wedge \omega$ defines a volume form on $M$. Thus, when we say a symplectic form, $n$ is assumed to be even. Integration along the subsets of $M$ defines a Lebesgue measure $m$. Indeed, by the para-compactness of $M$, locally $m$ is written as a product of a $\mathrm{C}^{1}$-function and the standard Lebesgue measure on $\mathbb{R}^{n}$ (via the $\mathcal{C}^{1}$-transition). This clarifies a Lebesgue measurable subset of $M$, a countable union of Lebesgue measurable subsets of $\mathbb{R}^{n}$ (via the $\mathcal{C}^{1}$-transition). Thanks to the well-known theory of Lebesgue measures and Borel measures, one guarantees any compact subset of $M$ is Lebesgue measurable and is of finite measure. By the compactness, the closed balls (with finite radii) are of finite measure, as well.

If one says $f$ preserves $\omega$, this means $f^{*} \omega=\omega$. When $\omega$ is a symplectic form, the $\omega$-preservation implies the volume-preservation. The volume-preservation of $f$ amounts to the measure-preservation. In the case, for a Lebesgue measurable subset $N \subset M$, we have $m(N)=m(f(N))$. 
We fix a manifold with metric $(X, d)$ and a homeomorphism $f: X \rightarrow X$. We define

$$
\mathcal{P}=\text { the set of } \mathbb{R}^{+} \text {-valued continuous functions on } X \text {. }
$$

Definition 2.1. A nonempty open subset $U$ of $X$ is an attractor block for $f$ if the closure of $f(U)$ is contained in $U$. When $U$ is an attractor block, the set

$$
A=\bigcap_{n \geq 0} \overline{f^{n}(U)}
$$

is called the weak attractor determined by $U$.

Definition 2.2. If $\varepsilon \in \mathcal{P}$, then $x_{0}, x_{1}, \cdots, x_{n}$ is an $\varepsilon$-chain if $d\left(f\left(x_{j}\right), x_{j+1}\right)<$ $\varepsilon\left(f\left(x_{j}\right)\right)$ for $0 \leq j<n-1$. The number $n$ is called the length of the $\varepsilon(x)$-chain. A point $p$ is strongly chain recurrent for $f$ if for every $\varepsilon \in \mathcal{P}$, there exists an $\varepsilon(x)$-chain of length at least 1 that begins and ends at $p$. We denote by

$$
C R^{+}(f)=\text { the set of all strong chain recurrence points of } f \text {. }
$$

Note that if $M$ is a compact manifold, then the strong chain recurrence point coincides with the usual chain recurrence point.

Definition 2.3. Let $U$ be an attractor block for $f$ and $A$ be the associated weak attractor. We define the basin of a weak attractor $A$ relative to $U, B(A ; U)$ as the open set $\cup_{n \geq 0} f^{-n}(U)$.

Every point of $B(A ; U)$ has the omega-limit sets contained in $A$. When $X$ is a compact space, $B(A ; U)$ is independent of $U$ while it is not true for non-compact manifolds. Therefore, we define the extended basin $B(A)$ of $A$ by the union of the sets $B(A ; U)$ as $U$ runs over all the absorbing sets that determine $A$.

\subsection{Strong chain recurrences of volume-preserving diffeomorphisms}

The chain recurrence theorem on compact manifolds with volume-preserving diffeomorphism is almost direct to prove. Our focus is non-compact manifolds. The simple examples below exhibit the failure of the chain recurrence theorem in the volume-preserving dynamics over non-compact manifolds.

Example 2.4. Let $M=\mathbb{R}$ and $f: M \rightarrow M$ given by $f(x)=x+1$. Then, $f$ preserves a differential form and no point of $M$ is a (strong) chain recurrence for $f$. While, let $U=(0, \infty)$, then we have that $U$ is an attractor block, with associated empty weak attractor.

Example 2.5. Let $M=\mathbb{R}^{2}$ and $f: M \rightarrow M$ given by $f(x, y)=(x+1, y)$. Let $\omega$ be a volume form (equivalently, a symplectic form) by $\omega=d x \wedge d y$. Then, it is clear that $f$ preserves $\omega$. Let

$$
U_{n}=\left\{(x, y) \in M \mid y<\frac{-1}{x-n}, x<n\right\} \cup\{(x, y) \in M \mid x \geq n\} .
$$


Since $f\left(U_{n}\right)=U_{n+1}$, we can easily check that $U_{0}$ is an attractor block for the translation $f$ and

$$
A=\{(x, y) \mid y \leq 0\}
$$

is the weak attractor determined by $U$. Whilst, no point of $M$ is a (strong) chain recurrence for $f$.

The following theorem by Hurley is a generalized version of Conley's theorem.

Theorem 2.6. $[5,6]$ If $X$ is a locally compact metric space and $f: X \rightarrow X$ is continuous, then the strong chain recurrence set $C R^{+}(f)$ of $f$ is the complement of the union of the set $B(A)-A$, as $A$ runs over the collection of weak attractors of $f$. I.e.,

$$
X-C R^{+}(f)=\bigcup_{\text {A:weak attractor }}(B(A)-A) .
$$

Here, the strong chain recurrence and weak attractors are defined with respect to a continuous map $f$ with a suitable adaptation of the definitions in the previous subsection.

The following proposition (and its corollary) shows the invariance of the weak attractors and the boundaries.

Proposition 2.7. Let $f$ be a homeomorphism on a metric space $X, U$ be an attractor block, and $A$ be an associated weak attractor. If a point $x$ is in $U-A$, then the intersection of the (positive) $f$-orbit of $x$ and $A$ is empty.

Proof. Let $O_{f}^{+}(x)$ be the (positive) $f$-orbit of $x$, i.e., $O_{f}^{+}(p)=\left\{f^{n}(p) \mid n \geq 0\right\}$. Suppose $O_{f}^{+}(x) \cap A \neq \varnothing$, Then there exists a nonnegative integer $k$ such that $f^{k}(x) \in$ $A$, that is, $f^{k}(x) \in \cap_{n \geq 0} \overline{f^{n}(U)}$. Note that, $f(\bar{U})=\overline{f(U)}$. Thus $x \in f^{n-k}(U)$ for all $n \geq 1$ and so $x \in \bar{A}$ by the shrinking property. This is a contradiction, which completes the proof.

When $f: X \rightarrow X$ is continuous, by the definition, it is easily shown that an attractor is positively $f$-invariant. If $f$ is a homeomorphism, an attractor $A$ is $f$-invariant, i.e., $f(A)=A$. Indeed, if $f(A) \neq A$ then there is an element $x$ in $A-f(A)$. From the definitions, $f^{-1}(x) \in U-A$, where $U$ is an associated attractor block. Then, Proposition 2.7, we must meet a contradiction. Hence an attractor is invariant.

Corollry 2.8. Let $f$ be a homeomorphism on a locally compact metric space $M$. Then the boundary of every weak attractor is positively $f$-invariant, that is, $f(\partial A) \subseteq \partial A$ for every weak attractor $A$.

Proof. Suppose the contrary of the conclusion. Then by the above statement, we may assume that there exists a boundary point $x$ satisfying $f(x)$ is in the interior of $A$. From the local compactness, we can choose compact neighborhood $C$ of $f(x)$ such that $f^{-1}(C)$ is also a compact neighborhood of $x$. Then, we are able to pick 
a point in $U-A$ where $U$ is an associated attractor block which determines $A$. By Proposition 2.7, it is a contradiction.

Now we embark on the main proposition and the main theorem for the (strong) chain recurrences on the non-compact manifolds. The proposition tells us that the points near an attractor with bounded orbits form a measure 0 set, in the volumepreserving dynamics. Recall that in Example, every orbit is unbounded thus the proposition trivially holds.

For $p \in M$, we denote $K^{+}(p):=\overline{O_{f}^{+}(p)}$. We call $M$ almost bounded for $f$, if for almost everywhere $p \in M, K^{+}(p)$ is compact. Since we are working on a metric space, the compactness of $K^{+}(p)$ amounts to the boundedness of $O_{f}^{+}(p)$.

Theorem 2.9. Let $M$ be a manifold with a volume form $\omega$, and $f$ be a volumepreserving diffeomorphism on $M$. If $M$ is almost bounded for $f$, then $M$ is strongly chain recurrent for $f$, i.e., every point of $M$ is strongly chain recurrent with respect to $f$.

Proof. We use Hurley's theorem (Theorem 2.6) for locally compact spaces. To prove our theorem, the nonexistence of weak attractors should be guaranteed. On the contrary, suppose that a nonempty proper weak attractor $A$ exists. Let $U$ be an associated attractor block of $A$ (so that $A \subsetneq U$ ). We will prove that the complement in $U-A$ of the set of points of $U-A$ with unbounded orbits is of measure 0 in the following proposition.

Proposition 2.10. Let $M$ be a manifold (not necessarily compact) with a volume form $\omega$. Let $f$ be a volume-preserving diffeomorphism on $M$. Let $A$ be any weak attractor and $U$ be an associated attractor block with $A \subsetneq U$. Then, the complement in $U-A$ of the set of points $p \in U-A$ with unbounded orbits is of measure 0 . That is, $m\left\{p \in(U-A) \mid O_{f}^{+}(p) \text { is unbounded }\right\}^{c}=0$, here $m$ is a measure induced by the volume form.

Proof. Let $p \in U-A$ and $K \subset U-A$ be a compact neighborhood of $p$ with a finite measure $c>0$. Let us fix any point $x_{0} \in M$. Let $B_{r}\left(x_{0}\right)$ be the closed ball of the radius $r$ centered at $x_{0} \in M$ (where $r \in \mathbb{Z}_{+}$). Let us define

$$
K_{r}=\left\{q \in K \mid f^{k}(q) \notin B_{r}\left(x_{0}\right) \text { for some positive integer } k\right\} .
$$

Note that

$$
K_{1} \supset K_{2} \supset K_{3} \supset \ldots
$$

and that

$$
L=\bigcap_{r \in \mathbb{Z}_{+}} K_{r}
$$

is the set of points of $K$ with unbounded orbits and $m(L)=m(K)$ implies the claim of the proposition. 
Now, to prove the proposition, it suffices to show that $L$ is of measure $c$. Let us observe

$$
\begin{aligned}
K-K_{r} & =\left\{q \in K \mid f^{k}(q) \in B_{r}\left(x_{0}\right) \text { for all } k \in \mathbb{Z}_{+}\right\} \\
& =\bigcap_{k \in \mathbb{Z}_{+}}\left\{q \in K \mid f^{k}(q) \in B_{r}\left(x_{0}\right)\right\}
\end{aligned}
$$

and thus $K-K_{r}$ is measurable as $\left\{q \in K \mid f^{k}(q) \in B_{r}\left(x_{0}\right)\right\}$ is measurable for each $k \in \mathbb{Z}+$. Therefore, $L$ is measurable as well.

We claim that

$$
m\left(\left(f^{k}(U)-f^{k}(A)\right) \cap B_{r}\left(x_{0}\right)\right) \rightarrow 0
$$

as $k \rightarrow \infty$. Indeed, Lebesgue's dominated convergence theorem assures it from the following:

(a) the definition of attractors (i.e., the descending sequence $U \supset f(U)$ $\left.f^{2}(U) \supset \ldots \supset A=\cap_{k} f^{k}(U)\right)$,

(b) the $f$-invariance of $A$,

(c) $f^{k}(U)-f^{k}(A)$ and $B_{r}\left(x_{0}\right)$ are measurable and their intersection is of finite measure.

Note that $\left\{q \in K \mid f^{k}(q) \in B_{r}\left(x_{0}\right)\right\}=f^{-k}\left(B_{r}\left(x_{0}\right)\right) \cap K$. Thus, we have

$$
\begin{aligned}
m\left(\left\{q \in K \mid f^{k}(q) \in B_{r}\left(x_{0}\right)\right\}\right) & =m\left(f^{-k}\left(B_{r}\left(x_{0}\right)\right) \cap K\right) \\
& =m\left(\left(B_{r}\left(x_{0}\right)\right) \cap f^{k}(K)\right)
\end{aligned}
$$

where the latter equality is due to the measure-preservation of $f$. Because of the inclusion $f^{k}(K) \subset f^{k}(U)-f^{k}(A)$ and (4), we obtain

$$
m\left(\left\{q \in K \mid f^{k}(q) \in B_{r}\left(x_{0}\right)\right\}\right) \rightarrow 0
$$

as $k \rightarrow \infty$ by Lebesgue's dominated convergence theorem. Therefore, for each $r$, we have $m\left(K-K_{r}\right)=0$, equivalently, $m\left(K_{r}\right)=m(K)-m\left(K-K_{r}\right)=c$. By applying Lebesgue's dominated convergence theorem to (3), we obtain $m(L)=c$, as desired.

Let us continue the proof of Theorem 2.9. By Proposition 2.10, almost every point of $U-A$ has an unbounded orbit. This contradicts to our assumption of the almost boundedness of $M$ with respect to $f$. This finishes the proof of Theorem 2.9 .

Recall that a riemannian manifold $M$ is said to be Lagrange-stable for a diffeomorphism $f$ if every closure of an orbit is compact, i.e., for each $p \in M, K^{+}(p)$ is a compact subset of $M$. Since the Lagrange-stability is a stronger condition than the almost-boundedness, we obtain the corollary. 
Corollry 2.11. Let $M$ be a manifold with a volume form $\omega$, and $f$ be a Lagrangestable volume-preserving diffeomorphism on $M$. Then, $M$ is strongly chain recurrent for $f$, that is, each point of $M$ is strongly chain recurrent with respect to $f$.

\section{References}

[1] M. Arnaud, C. Bonatti and S. Crovisier, Dynamiques symplectiques génériques, Ergodic Theory Dynam. Systems, 25(2005), 1401-1436.

[2] J. Choy and H.-Y. Chu, On the Envelopes of Homotopies, Kyungpook Math. J., 49(3)(2009), 573-582.

[3] J. Choy, H.-Y. Chu and M. Kim, Volume preserving dynamics without genericity and related topics, Commun. Korean Math. Soc., 27(2012), 369-375.

[4] C. Conley, Isolated invariant sets and the morse index, C. B. M. S. Regional Lect., 38(1978).

[5] M. Hurley, Chain recurrence and attraction in noncompact spaces, Ergodic Theory Dynam. Systems, 11(1991), 709-729.

[6] M. Hurley, Noncompact chain recurrence and attraction, Proc. Amer. Math. Soc., 115(1992), 1139-1148. 